\title{
Diffusion, convection, and solidification in cw-mode free electron laser nitrided titanium
}

\author{
Daniel Höche, ${ }^{1, a)}$ Michelle Shinn, ${ }^{2}$ Sven Müller, ${ }^{1}$ and Peter Schaaf ${ }^{3, b)}$ \\ ${ }_{1}^{1}$ II. Physikalisches Institut, Universität Göttingen, Friedrich-Hund-Platz, 1, 37077 Göttingen, Germany \\ ${ }^{2}$ Free Electron Laser Group, Thomas Jefferson National Accelerator Facility, Newport News, \\ Virginia 23606, USA \\ ${ }^{3}$ Institut für Werkstofftechnik, FG Werkstoffe der Elektrotechnik, TU Ilmenau, Postfach 100565 , \\ 98684 Ilmenau, Germany
}

(Received 21 January 2009; accepted 8 February 2009; published online 16 April 2009)

\begin{abstract}
Titanium sheets were irradiated by free electron laser radiation in cw mode in pure nitrogen. Due to the interaction, nitrogen diffusion occurs and titanium nitride was synthesized in the tracks. Overlapping tracks have been utilized to create coatings in order to improve the tribological properties of the sheets. Caused by the local heating and the spatial dimension of the melt pool, convection effects were observed and related to the track properties. Stress, hardness, and nitrogen content were investigated with x-ray diffraction, nanoindention, and resonant nuclear reaction analysis. The measured results were correlated with the scan parameters, especially to the lateral track shift. Cross section micrographs were prepared and investigated by means of scanning electron microscopy. They show the solidification behavior, phase formation, and the nitrogen distribution. The experiments give an insight into the possibilities of materials processing using such a unique heat source. (C) 2009 American Institute of Physics. [DOI: 10.1063/1.3097781]
\end{abstract}

\section{INTRODUCTION}

Nitriding of metals such as $\mathrm{Fe}, \mathrm{Al}$, and $\mathrm{Ti}$ is a well known possibility to improve the material properties such as microhardness and wear resistance of strained technical surfaces. ${ }^{1}$ Established methods for such surface treatments are plasma or gas nitriding known as physical vapor deposition (PVD), ${ }^{2}$ the classical chemical vapor deposition, and salt bath nitriding. Alternatively, it is possible to modify the metallic surface with laser radiation and to synthesize hard coatings directly ${ }^{3}$ in a reactive ambient. Previous investigations have shown the successful synthesis of titanium nitride (TiN) coatings with laser irradiation at different wavelengths ${ }^{4-8}$ also using a free electron laser (FEL). ${ }^{9-11}$ The following investigation shows the possibilities of nitriding by means of cw-mode FEL irradiation. This method offers many advantages in relation to the mentioned techniques. There is a reduction in the processing time and an effective surface treatment due to the high power micropulses at high repetition rates. Contrarily, intrinsic stress, microstrain, and inhomogeneity remain the main problems. Also the lattice orientation of the cubic titanium nitride is a decisive factor for the technical quality of coatings produced by using overlapping tracks. Texture influences strain development and eventually crack formation and propagation, which leads to a strong decrease in quality. First investigations on texture and strain development in relation to the process parameters have been reported in the literature. ${ }^{12-14}$

This work resolves the coating properties of TiN synthesized in nitrogen ambient with overlapping tracks at different lateral shifts $\delta$. Using different kinds of characterization

\footnotetext{
a)Electronic mail: dhoeche@gwdg.de.

${ }^{b)}$ Electronic mail: peter.schaaf@tu-ilmenau.de.
}

methods such as scanning electron microscopy (SEM), nanoindention, resonant nuclear reaction analysis (RNRA), and $\mathrm{x}$-ray diffraction (XRD) offers an insight into the physics of the treatments, the basic processes, and the possibilities for optimization. The FEL will be valuated for his suitability to be a tool for materials treatment.

\section{EXPERIMENTS}

\section{A. Sample treatment}

Titanium sheets (uncoated, $1 \mathrm{~mm}$ thickness, purity $>99.98 \%$ ) were cut into pieces of $15 \times 15 \mathrm{~mm}^{2}$ size. For the FEL treatments, the samples were placed in a modified chamber, first evacuated to $10^{-6}$ atm and then filled with nitrogen (purity $99.999 \%$ ) to a pressure of $0.115 \mathrm{MPa}$.

The focused beam reached the sample surface through a fused silica window. In order to treat the whole surface of the samples and to synthesize coatings, the chamber was mounted onto a computer-controlled $x-y$ table. A relative velocity $v=24 \mathrm{~mm} / \mathrm{s}$ in the $x$-direction was used. Figure 1

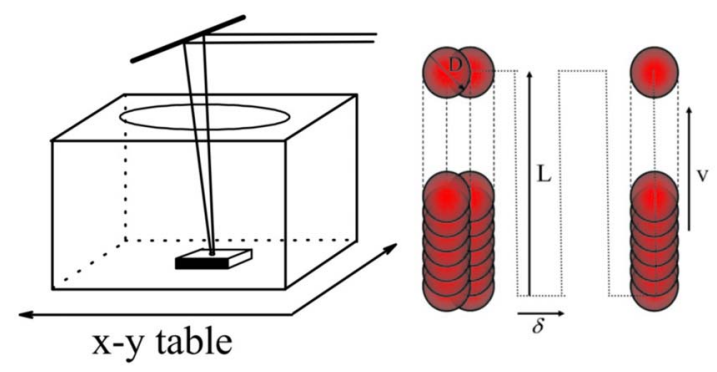

FIG. 1. (Color online) Experimental and scanning scheme of the nitriding treatment using the spot size $D_{B}$, the scan length $L$, the lateral shift $\delta$, and the scan velocity $v$. 
TABLE I. FEL beam and scan parameters during the experiments at 0.115 $\mathrm{MPa}$ nitrogen background pressure.

\begin{tabular}{lcc}
\hline \hline Parameter & Symbol & Value \\
\hline Average intensity & $I_{0}$ & $3 \times 10^{9} \mathrm{~W} / \mathrm{m}^{2}$ \\
Average power & $P$ & $655 \mathrm{~W}$ \\
Maximum intensity micro & $I_{0_{\text {mic }}}$ & $2.2 \times 10^{15} \mathrm{~W} / \mathrm{m}^{2}$ \\
Pulse energy & $E_{\text {mic }}$ & $125 \mu \mathrm{J}$ \\
Pulse frequency & $f_{(\text {mic })}$ & $4.68 \mathrm{MHz}$ \\
Beam diameter FWHM & $D_{B}$ & $600 \mu \mathrm{m}$ \\
Pulse duration micro & $\tau_{\text {mic }}$ & $200-400 \mathrm{fs}$ \\
Scan velocity & $v$ & $2.4 \mathrm{~cm} / \mathrm{s}$ \\
Scan length & $L$ & $1 \mathrm{~cm}$ \\
\hline \hline
\end{tabular}

shows the processing scheme. The experiments have been performed at the FEL-user facility at $\mathrm{cw}$ mode. This mode operates with continuous micropulse trains of subpicosecond pulses $\left(\tau_{\text {mic }}=200-400 \mathrm{fs}\right)$ at a frequency $f_{(\mathrm{mic})}$ of 4.68 $\mathrm{MHz} .{ }^{15,16}$ By means of specific optics the raw beam of about $6 \mathrm{~cm}$ in diameter was redirected to the lens and focused to the used spot size $D_{B}$. Additionally a cam was installed for monitoring the treatments in the safety area. Table I summarizes the used parameters.

\section{B. Analysis}

The synthesized TiN coatings have been investigated with several methods. Micrographs have been realized by means of SEM, which was performed with a LEO Supra 35 Gemini, whereas all cross section images were recorded with a quadral backscattering detector.

In order to get information about the mechanical properties the microhardness was measured with a nanoindenter (Fischerscope HV100). ${ }^{3}$ It operates with a Vickers diamond tip and a maximum force of $1 \mathrm{~N}$. Because of the surface roughness and their strong influence on the method, the measurements were done at the cross sections.

One of the most interesting results after the treatments is the distribution of $\mathrm{N}$ atoms. Nitrogen depth profiling was carried out by means of the resonant nuclear reaction analysis ${ }^{17}$ employing the reaction ${ }^{15} \mathrm{~N}(p, \alpha \gamma){ }^{12} \mathrm{C}$. The measurements were performed at the Göttingen IONAS (Ref. 18) accelerator. Details are given in Ref. 3. The nitrogen depth profiles were analyzed assuming diffusion profiles in first approximation.

The microstructure of the layers was analyzed by XRD in grazing incidence (GIXRD) and Bragg-Brentano $(\Theta-2 \Theta)$ geometry, using a Bruker AXS diffractometer equipped with a $\mathrm{Cu} K \alpha$ tube and a thin film attachment. GIXRD yielded the nitrogen content at the information depth and using the $\sin ^{2} \Psi$ method, ${ }^{19}$ it gives quantified data about the intrinsic stress. $\Theta-2 \Theta$ scans allow to perform peak analysis and to determine the grain size and the microstrain with methods like Williamson-Hall plot (WHP) or Warren-Averbach (WA) analysis. $^{20,21}$

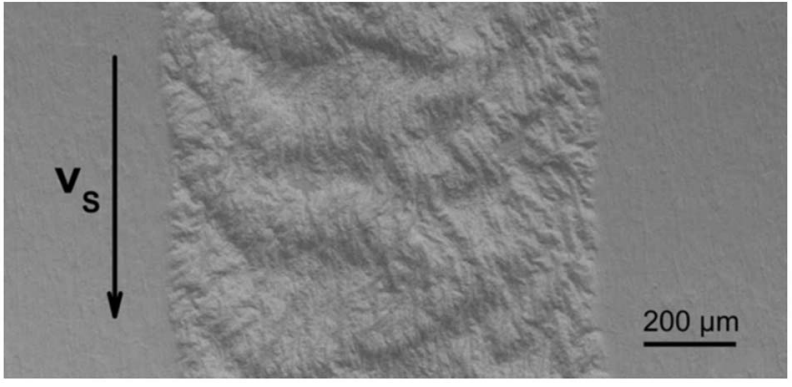

FIG. 2. SEM: Top view on a solidified single TiN track. Convectional flow induced surface deformation could be observed and also structures with a short roughness spacing (oscillations).

\section{RESULTS}

\section{A. Surface}

After irradiation, the titanium sheets are gold-colored and inhomogeneous tracks developed along the scan axis. During the synthesis a strong reaction of the nitrogen with the liquid titanium was observed. The titanium nitride formed almost exclusively within the molten range, which refers to a strong difference in the diffusion coefficients $D_{\mathrm{Ti}}$ of the $\mathrm{N}$ atoms into the different conditions of aggregation of the titanium. In the literature, ${ }^{22-24}$ values of $D_{\mathrm{Ti}}$ vary between the liquid and solid phases around nearly four orders of magnitude. The top view on the solidified tracks is represented in Fig. 2. They exhibit a higher roughness compared to the untreated titanium samples and depend on the scan velocity, respectively, laser-material interaction time. The reduction in the surface quality is a result of strong convectional flow in the melt pool during the synthesis, caused by the high Prandtl number $(0.11)$ of liquid titanium, ${ }^{25}$ which makes it sensitive for capillary flow. Due to high temperature gradients and strong evaporation, Marangoni convection and also the recoil pressure force lead to a strong melt flow and have to be optimized in relation to technical surfaces.

"Humps" and melt injection from the tracks were not observed; however, a periodic structure has been developed, which is a result of the melt flow behavior and the acting surface forces (surface tension). Short-wavelength periodical structures could be observed too. Due to induced shock waves, oscillations on the melt occur. They are also known as Rayleigh-Taylor instabilities. The solidified surfaces are free of cracks which is essential for wear resistance. Due to the moderate heat entry caused by the scan velocity and the temporal beam structure (pulse trains), thermal stress keeps small.

\section{B. Nanoindention}

In order to quantify the changes in the mechanical characteristics of the system, the microhardness was measured at several points. Since the surface finish of the nitrified samples is rather bad (roughness) and thus the measurements are strongly affected, nanoindention has been performed in the transverse cross section. In addition the samples were polished and adjusted at the measuring system. Figure 3 shows measured hardness profiles at the dendritic solidified TiN, at the interspaces, and at the substrate. 


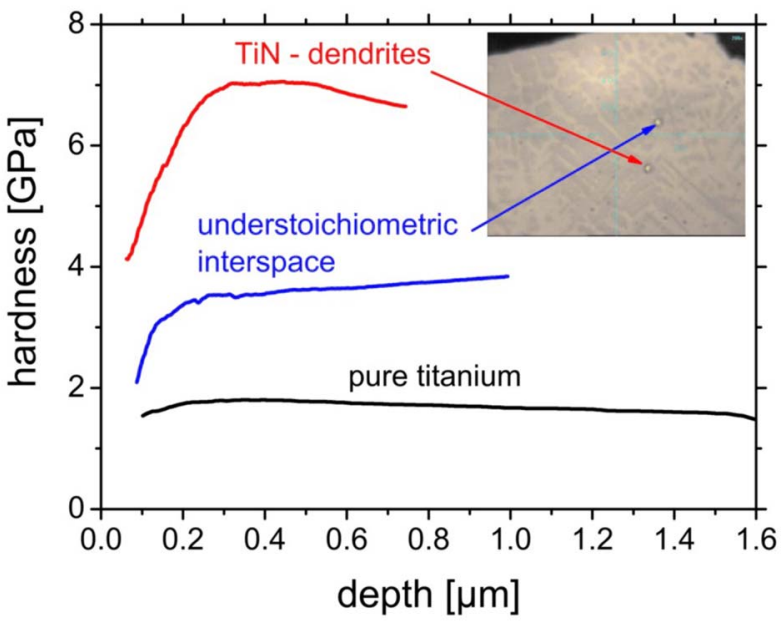

FIG. 3. (Color online) Hardness at different regions (dendrites, interspaces, and substrate) of cw-mode nitrided titanium.

It is shown that one must regard different ranges of the sample. TiN dendrites close to the surface contain stoichiometric TiN and their hardness amounts to over $7 \mathrm{GPa}$. In relation to the improvement of mechanical properties, the hardness has been increased around a factor of 5 compared to the substrate. Between the dendrites, domains with lower nitrogen content exist, which consist of understoichiometric $\mathrm{TiN}_{x}$ and nitrogen in solution in $\alpha$-Ti (solubility $\mathrm{N}-\mathrm{Ti}$ of 23 at. \%). ${ }^{26}$ Here, a hardness of approximately $4 \mathrm{GPa}$ was measured. In order to get information about the hardness depth profile, a line scan was accomplished along the surface toward the substrate shown in Fig. 4.

The shape of the curve resembles a diffusion profile. Close to the surface the hardness (about $7 \mathrm{GPa}$ ) is very high and drops down in the areas with less nitrogen (melt pool ground-4 $\mathrm{GPa}$ ). At least the hardness reaches the value of the pure $\alpha$-titanium (approximately $1.5 \mathrm{GPa}$ ). The region of influence of the nitrogen corresponds to a depth of approximately $250 \mu \mathrm{m}$, which is close to the melting depth. Due to the diffusionlike profile there is no exact demarcation between the coating and the substrate. This makes it difficult to

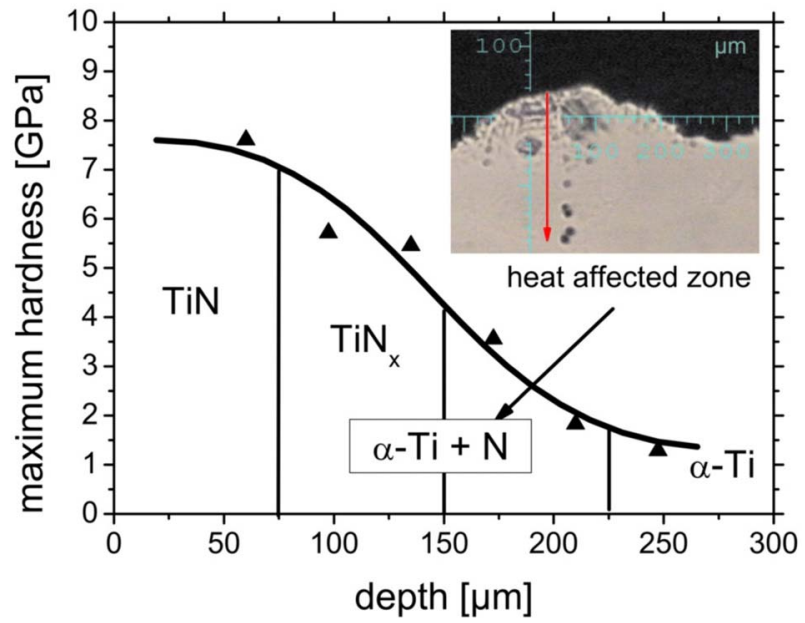

FIG. 4. (Color online) Hardness depth profile by means of a line scan (along the arrow in the inset). The values are consistent with the maximum hardness according to Fig. 3.

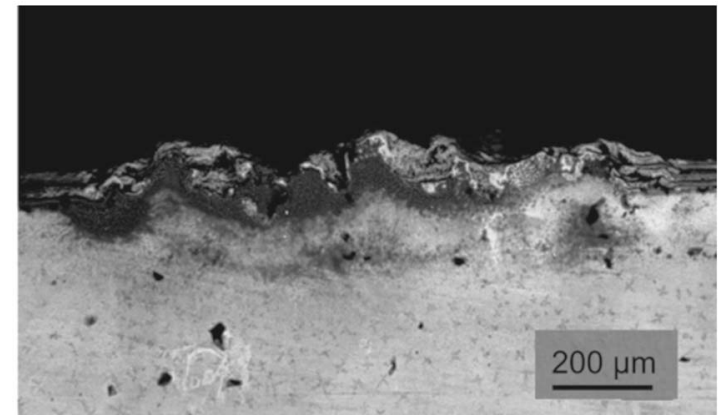

FIG. 5. SEM: Cross section micrograph of a single transversal track. Black regions correspond to a high nitrogen content.

indicate an accurate coating thickness. It is most meaningful to define the coating thickness only at the range where cubic TiN is present and where significantly changes in the mechanical characteristics are obtained. A depth of approximately $150 \mu \mathrm{m}$ was measured. Such a coating thickness is enormous compared with other manufacturing processes (PVD-some microns). ${ }^{27}$ However, these values come together with disadvantages such as inhomogeneities and roughness.

\section{Nitrogen depth profiling}

A SEM cross section is shown in Fig. 5 in the transverse direction. The micrograph was taken with a backscattering detector so that black areas in the track correspond to nitrogen-rich phases. Besides a further problem becomes recognizably, the inhomogeneity. It becomes clear that the incorporation of the $\mathrm{N}$ atoms into the Ti-matrix runs unordered. The reason for that behavior is the strong competition between convective and diffuse material transport. Next a nitrogen-poor range within the lower region of the melt pool is observable. There are understoichiometric phases and also nitrogen in solution in titanium due to the high solubility of 23 at. \%. Below the nitrogen interaction zone the heat affected zone could be observed to a depth of approximately $300 \mu \mathrm{m}$ (according Fig. 4).

In order to quantify the nitrogen content, RNRA was performed at three samples with different lateral shifts $\delta$ of $0.4,0.6$, and $0.8 \mathrm{~mm}$. The proton beam diameter during the measurements was $3 \mathrm{~mm}$, whereby information about the average nitrogen distribution becomes available. Figure 6 shows the $\mathrm{N}$ content of the samples in the range near the surface $(<600 \mathrm{~nm})$. It becomes clear that for the two samples with $\delta$ smaller than the focus diameter $D(\delta<D)$, a closed stoichiometric titanium nitride coating could be observed. With the $0.8 \mathrm{~mm}$ sample the loss of the concentration results from the unmelted ranges between the individual tracks. The nitrogen content is lower close to the surface and increases during the first $100 \mathrm{~nm}$ to its maximum value. In order to receive a more exact insight in the process it is necessary to quantify the transport of the nitrogen.

A mathematical description of these depth profiles can be given using the complementary error function (solutions of the diffusion equation) by a superposition of two profiles. $^{28}$ The solution, as shown in Eq. (1), contains two diffusion lengths, a long range one (index 1) and one in the 


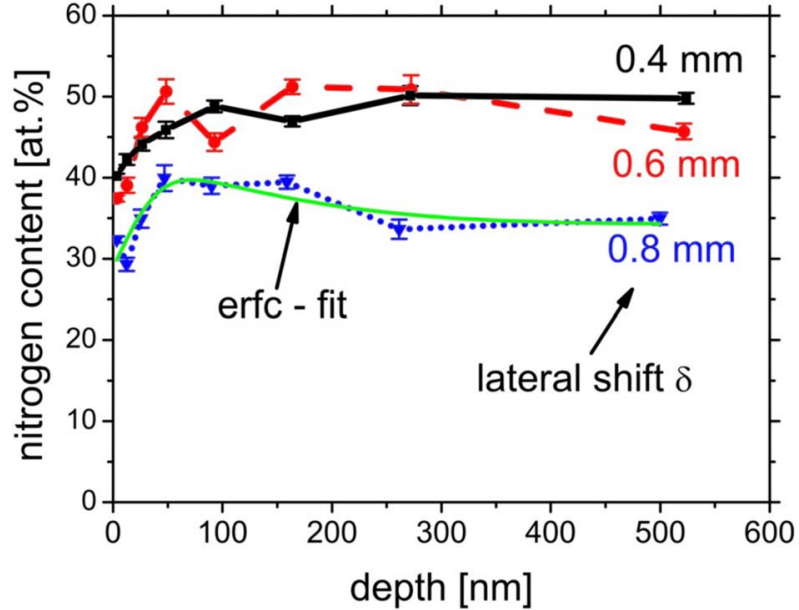

FIG. 6. (Color online) Nitrogen depth profiling by means of RNRA at cwmode nitrided titanium synthesized with different shifts $\delta$ from 0.4 to 0.8 $\mathrm{mm}$. An example for an error function fit is shown too.

short range (index $s$ ). As next it is assumed that there are three concentrations $c_{0}, c_{l}$, and $c_{s}$, which limit the respective ranges. The diffusion profile at the long range mainly describes macroscopic nitrogen diffusion as well as the coating thickness and it determines the macroscopic characteristics. Further it contains information about the convectional nitrogen transport induced by Marangoni forces. In contrast to it, short range diffusion describes the effects near the surface and it contains information about the absorption of nitrogen and its incorporation and about oxidation and contamination effects too.

$$
\begin{aligned}
c= & c_{0}+c_{l}\left[1-\operatorname{erf}\left(\frac{x}{\sqrt{4 D_{\mathrm{Ti}} t_{l}}}\right)\right] \\
& +\left(c_{s}-c_{0}\right)\left[1-\operatorname{erf}\left(\frac{x}{\sqrt{4 D_{\mathrm{Ti}} t_{s}}}\right)\right] .
\end{aligned}
$$

The parameter describing the kinetics of the process is the diffusion lengths $L_{l, s}$ which determine the characteristics of the coatings. By using the well known relation of the diffusion processes $L_{l, s}=\sqrt{4} D_{\mathrm{Ti}} t_{l, s}$ it is possible to obtain information about the diffusion times $t_{l, s}$. Table II summarizes the analysis results.

Unfortunately, the information depth of the method is limited to approximately $500 \mathrm{~nm}$ due to the proton energy; thus only qualitative statements about the long range diffusion can be made. As shown in Fig. 6 the diffusion length exceeds these $500 \mathrm{~nm}$, which correlates with the other observations. By means of an assumption of an average nitrogen diffusion coefficient $D_{\mathrm{Ti}}$ in the titanium melt pool of approximately $2 \times 10^{-4} \mathrm{~cm}^{2} / \mathrm{s}$ according to previous works, ${ }^{29}$ it is

TABLE II. Diffusion data of cw-mode nitrided titanium.

\begin{tabular}{cccccc}
\hline \hline Sample & $\begin{array}{c}\delta \\
(\mathrm{mm})\end{array}$ & $\begin{array}{c}L_{l} \\
(\mathrm{~nm})\end{array}$ & $\begin{array}{c}t_{l} \\
(\mathrm{~ms})\end{array}$ & $\begin{array}{c}L_{s} \\
(\mathrm{~nm})\end{array}$ & $\begin{array}{c}t_{s} \\
(\mathrm{~ns})\end{array}$ \\
\hline 1 & 0.4 & $>4000$ & $>0.2$ & 49 & 30 \\
2 & 0.6 & $>3500$ & $>0.13$ & 33 & 14 \\
3 & 0.8 & $>3000$ & $>0.1$ & 41 & 21 \\
\hline \hline
\end{tabular}

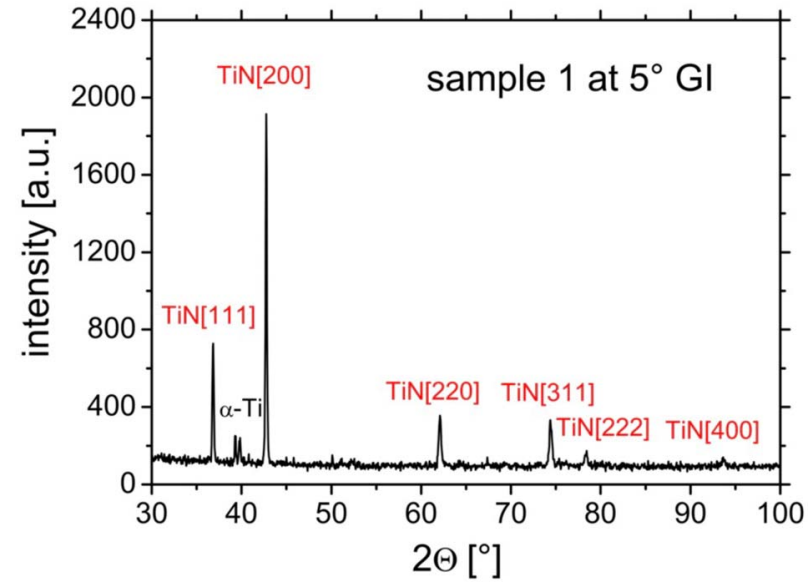

FIG. 7. (Color online) GIXRD: Diffraction pattern of sample 1 at $5^{\circ}$.

possible to determine diffusion times $t_{l}, s$. In the case of long range diffusion times of some hundred microseconds have been measured. This conforms to the melt duration during the scan. Since the profiles do not provide any information from deeper ranges, it is impossible to give more accurate statements. The investigations of the profiles close to the surface show a nitrogen rise at a diffusion length of approximately $40 \mathrm{~nm}$. They are contaminations and their effects are not subject of these studies.

\section{XRD}

Intrinsic stress and phase analysis. GIXRD offers many possibilities of analysis. Using $5^{\circ}$ grazing incidence and measuring $2 \Theta$ over a range of $30^{\circ}-100^{\circ}$, the three samples have been analyzed. Figure 7 shows the measured diffraction pattern of an example.

All measurements exclusively show $\delta$-TiN, whereby all appropriate reflexes are indicated in Fig. 7. The other reflexes correspond exclusively to the substrate material of the hexagonal $\alpha$-titanium. By means of GI measurements one can determine the ratio of the phases to each other with Vegard's law. The areas below the reflexes correlate linearly with the chemical composition of the coating within the range of the information depth of the measurement. This amounts to approximately $2 \mu \mathrm{m}$ in the present case. For the two samples with lateral shifts $\delta$ of 0.4 and $0.6 \mathrm{~mm}$ the portion of TiN exceeds $95 \%$ within these $2 \mu \mathrm{m}$. In regard to the sample with a $\delta$ of $0.8 \mathrm{~mm}$ it is an important fact that the lateral shift was bigger than the focus diameter. In this case one receives a portion of approximately $60 \%$ TiN. Moreover, the GI measurements offer the possibility to measure intrinsic stress. By means of the $\sin ^{2} \Psi$ procedure the stress in the coatings has been determined and compared.

TABLE III. Stress in the samples according to the $\sin ^{2} \Psi$ procedure.

\begin{tabular}{ccc}
\hline \hline Sample & $\begin{array}{c}\delta \\
(\mathrm{mm})\end{array}$ & $\begin{array}{c}\sigma / E \\
(\%)\end{array}$ \\
\hline 1 & 0.4 & 4.2 \\
2 & 0.6 & 2.5 \\
3 & 0.8 & 3.4 \\
\hline
\end{tabular}




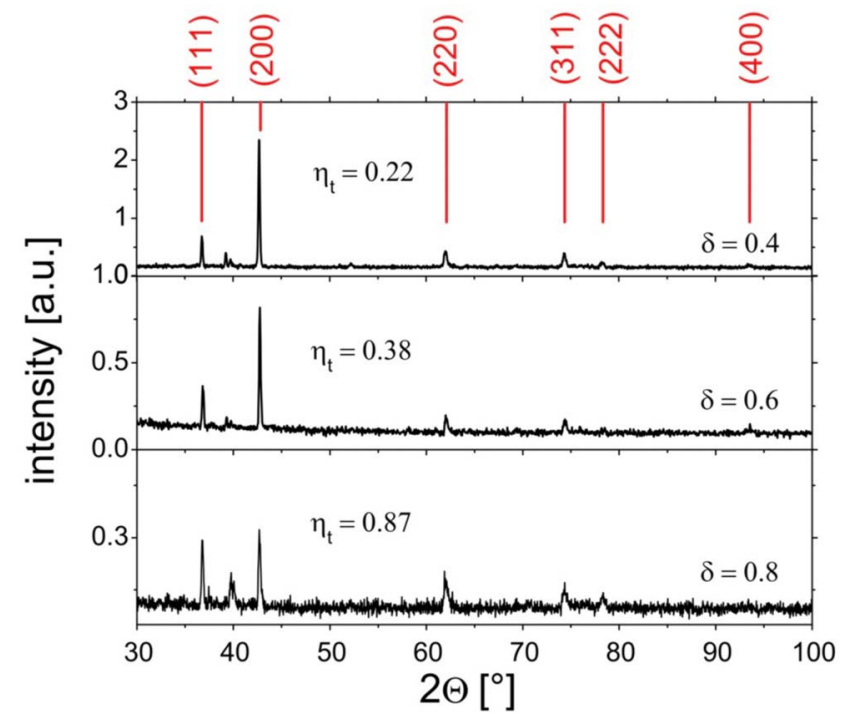

FIG. 8. (Color online) $\Theta-2 \Theta$ : Diffraction pattern of the three samples. $\eta_{t}$ describes the texture parameter.

As represented in Table III, the stress in the system corresponds to approximately $3 \%$ of the elastic modulus. The determination of absolute values of $\sigma$ is very difficult. The Poisson ratio $(\approx 0.25)$ and the elastic modulus are strongly coupled to the nitrogen content and varying in the literature. The influence of the stress becomes strong as one sees at the curvature of the treated sheets.

Lattice properties: strain, grain size, and texture. In order to determine the grain characteristics it is necessary to measure the diffraction pattern in Bragg-Brentano geometry. This enables the possibility to receive information about grain sizes $D$ and the lattice strain $\varepsilon$ in the TiN coatings. Moreover, the lattice orientation could be observed. A texture parameter $\eta_{t}$ was defined, which indicates the ratio of the maximum intensities of the (111) to (200) reflex $\left[\eta_{t}\right.$ $=I(111) / I(200)] . \eta_{t}$ decreases with a rising intensity of the (200) reflex, which leads to the following physical sense: if $\eta_{t}=0$ one has a perfect (200) texture of the lattice, but for $\eta_{t}=0.72$ it is an ideal polycrystal. In Fig. 8 the $\Theta-2 \Theta$-diffraction pattern and orientations are shown.

With an increase in energy entry (smaller $\delta$ ) the titanium nitride lattice is solidified stronger in the (200) direction. For sample 1 with the strongest texture, additionally a pole figure was measured and shown in Fig. 9. It verifies the (200) alignment of the atomic planes and the development of a fiber texture.
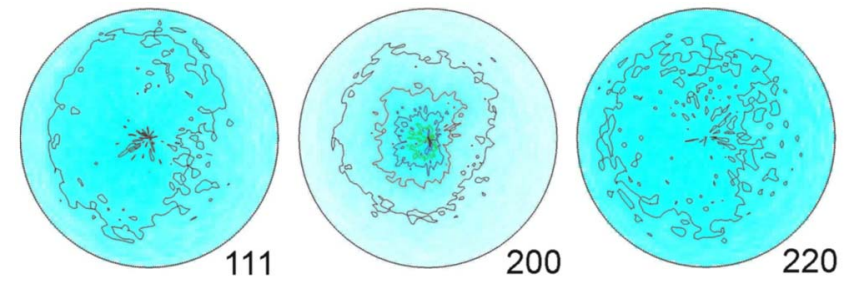

FIG. 9. (Color online) Pole figures of the three most important reflexes of cubic titanium nitride measured at sample 1 . The (200) orientation determines the measurement.

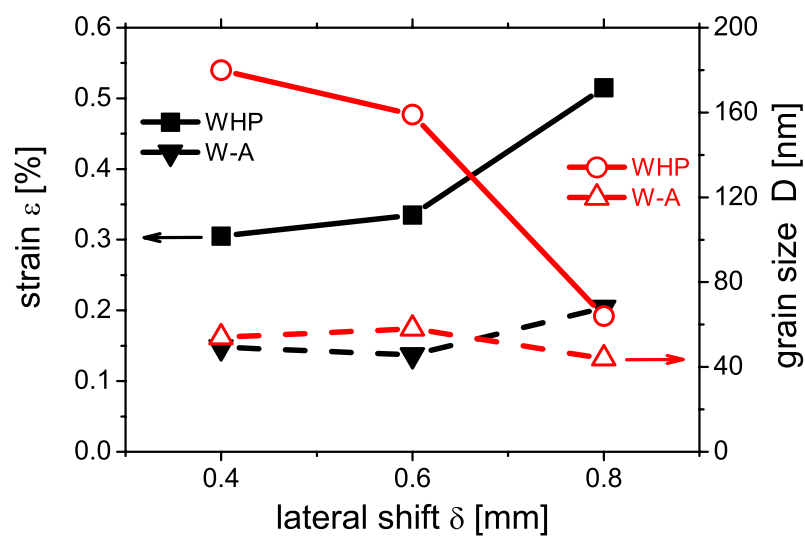

FIG. 10. (Color online) Grain size $D$ and lattice strain $\varepsilon$ in relation to the lateral shift $\delta$ determined by means of WHP and WA analyses.

The grain size was determined with the well known methods WHP and WA. The results are represented in Fig. 10. A rising energy entry leads to an increase in the grain size and in contrast, the lattice strain decreases. This behavior is based on the time for phase formation and the nitrogen content. In the case of sample 1 , the conditions of formation are most favorable.

The different absolute values between the two procedures WHP and WA result from the theoretical ansatz of the methods. While the WHP indicates the maximum values, the WA analysis determines the weighted means. ${ }^{30,31}$ Detailed information are available in the literature. ${ }^{20,21}$

Figure 11 confirms the texture development. Dendritic solidified TiN at the track edges shows a weak alignment perpendicular to the surface. This corresponds to the redirected lattice planes. This solidification behavior seems to be independent of convectional induced surface deformation and determines the macroscopic properties of the sheets. Additionally, a martensitic transformation (hcp- $\alpha^{\prime}$ titanium) in the heat affected zone below the dendrites was observed. It results from the rapid cooling in the melt due to high temperature gradients.

\section{CONCLUSIONS}

The experiments show the possibilities of direct laser synthesis of functional coatings in a reactive ambient using a

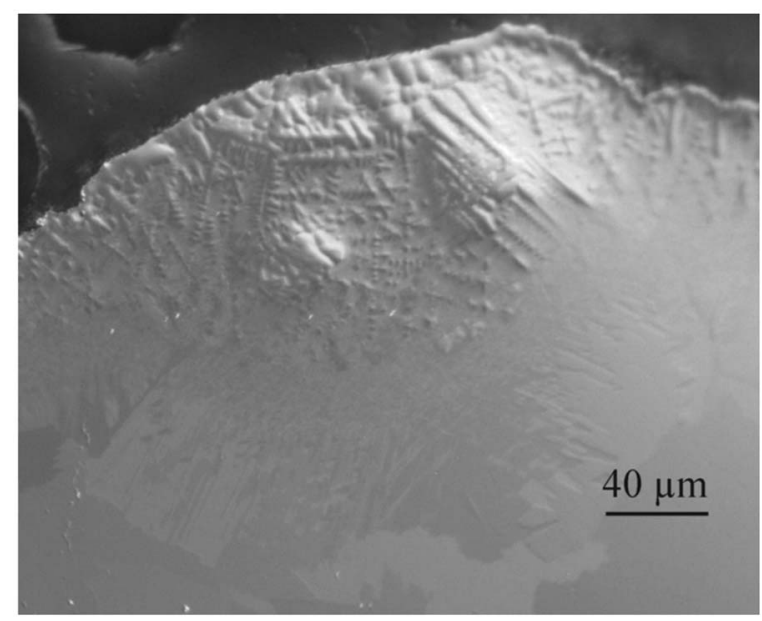

FIG. 11. SEM of solidified TiN dendrites at the track edges. A weak alignment perpendicular to the surface could be observed [weak (200) texture]. 
high power FEL at cw mode. Coating thicknesses of about $100 \mu \mathrm{m}$ with varying nitrogen contents and dendritic growth morphologies were observed. They were limited by the melting depth. The surface characteristics are governed by track overlapping, convectional flow, and oscillations. A hardness of about $8 \mathrm{GPa}$ was measured for stoichiometric TiN. This result corresponds to an increase in wear resistance and an improved friction behavior. Cross section micrographs and investigation of nitrogen distributions show the formation of understoichiometric $\mathrm{TiN}_{x}$ phases and soluted $\mathrm{N}$ atoms in pure titanium. XRD reveals an intrinsic stress of $4 \%$ of elastic modulus and shows the development of a (200) fiber texture for increasing heat entry. Lattice strain $(0.5 \%)$ and grain sizes of about $100 \mathrm{~nm}$ have been developed during the treatments.

These results give an insight into the possibilities of materials processing using such a unique heat source and in the modifications of materials under extreme conditions. Due to the pulse trains the nitriding conditions above the surface are stable, which leads to effective dissociation and optimal absorption. The thickness of the nitrided region is similar to $\mathrm{CO}_{2}$ laser nitrided titanium ${ }^{32}$ and much higher in comparison to Nd:YAG experiments. ${ }^{33,34}$ In relation to technical surfaces, the process still needs optimization and further investigations.

\section{ACKNOWLEDGMENTS}

This work is supported by the Deutsche Forschungsgemeinschaft under Grant No. DFG Scha 632/4. The Jefferson Laboratory is supported by the U.S. Dept. of Energy, the Office of Naval Research, the Commonwealth of Virginia, and the Laser Processing Consortium. Kevin Jordan and Joseph F. Gubeli III are gratefully acknowledged for their assistance at the FEL.

\footnotetext{
${ }^{1}$ A. Bloyce, P. H. Morton, and T. Bell, Surface Engineering of Titanium and Titanium Alloys (ASM International, Materials Park, OH, 1994).

${ }^{2}$ H. C. Man, Z. D. Cui, T. M. Yue, and F. T. Cheng, Mater. Sci. Eng., A 355, 167 (2003).

${ }^{3}$ P. Schaaf, Prog. Mater. Sci. 47, 1 (2002).

${ }^{4}$ H. Xin, S. Mridha, and T. N. Baker, J. Mater. Sci. 31, 22 (1996).

${ }^{5}$ S. Katayama, A. Matsunawa, A. Morimoto, S. Ishimoto, and Y. Arata,
}

Proceedings of the Third International Colloquium on Welding and Melting by Electron and Laser Beam, Lyon, France, September 1983 (unpublished).

${ }^{6}$ A. Kloosterman, Surface Modification of Titanium With Lasers (Groningen University Press, Groningen, 1998)

${ }^{7}$ A. Kloosterman and J. De Hosson, Scr. Metall. Mater. 33, 567 (1995).

${ }^{8}$ A. Kloosterman and J. De Hosson, J. Mater. Sci. 32, 6201 (1997).

${ }^{9}$ E. Carpene, M. Shinn, and P. Schaaf, Appl. Phys. A: Mater. Sci. Process. 80, 1707 (2005).

${ }^{10}$ D. Höche, M. Shinn, J. Kaspar, G. Rapin, and P. Schaaf, J. Phys. D: Appl. Phys. 40, 818 (2007).

${ }^{11}$ D. Höche, G. Rapin, J. Kaspar, M. Shinn, and P. Schaaf, Appl. Surf. Sci. 253, 8041 (2007).

${ }^{12}$ U. Oh and J. Je, J. Appl. Phys. 74, 1692 (1993).

${ }^{13}$ J. Pelleg, L. Zevin, S. Lungo, and N. Croitoru, Thin Solid Films 197, 117 (1991).

${ }^{14}$ P. Patsalas, C. Gravalidis, and S. Logothetidis, J. Appl. Phys. 96, 6234 (2004).

${ }^{15}$ S. Benson, G. Biallas, J. Boyce, D. Bullard, J. Coleman, D. Douglas, F. Dylla, R. Evans, P. Evtushenko, and A. Grippo, Nucl. Instrum. Methods Phys. Res. A 582, 14 (2007).

${ }^{16}$ M. D. Shinn, Proc. SPIE 4065, 434 (2000).

${ }^{17}$ F. Landry and P. Schaaf, Nucl. Instrum. Methods Phys. Res. B 179, 262 (2001).

${ }^{18}$ M. Uhrmacher and H. Hofsäss, Nucl. Instrum. Methods Phys. Res. B 240, 48 (2005).

${ }^{19}$ P. Prevey, Metals Handbook (ASM International, Metals Park, OH, 1986).

${ }^{20} \mathrm{G}$. Williamson and W. Hall, Acta Metall. 1, 22 (1953).

${ }^{21}$ B. Warren, X-Ray Diffraction (Dover, New York, NY, 1990).

${ }^{22}$ E. Fromm and E. Gebhardt, Gases and Carbon in Metals (Zentralstelle für Atomkernenergie-Dokumentation, Eggenstein-Leopoldshafen, 1978).

${ }^{23}$ F. W. Wood and O. G. Paasche, Thin Solid Films 40, 131 (1977).

${ }^{24}$ A. Anttila, J. Räisänen, and J. Keinonen, Appl. Phys. Lett. 42, 498 (1983).

${ }^{25}$ A. Robert and T. Debroy, Metall. Mater. Trans. B 32, 941 (2001).

${ }^{26}$ H. Wriedt and J. Murray, J. Phase Equilib. 8, 378 (1987).

${ }^{27}$ W. Sproul, Surf. Coat. Technol. 81, 1 (1996).

${ }^{28}$ P. Schaaf, F. Landry, and K. Lieb, Appl. Phys. Lett. 74, 153 (1999).

${ }^{29}$ D. Höche, H. Schikora, H. Zutz, A. Emmel, R. Queitsch, and P. Schaaf, J. of Coat. Tech. and Res. 5, 505 (2008).

${ }^{30}$ D. Höche, H. Schikora, H. Zutz, R. Queitsch, A. Emmel, and P. Schaaf, Appl. Phys. A: Mater. Sci. Process. 91, 305 (2008)

${ }^{31}$ H. Savaloni, M. Gholipour-Shahraki, and M. Player, J. Phys. D: Appl. Phys. 39, 2231 (2006)

${ }^{32}$ M. Raaif, F. El-Hossary, N. Negm, S. Khalil, A. Kolitsch, D. Höche, J. Kaspar, S. Mandl, and P. Schaaf, J. Phys. D: Appl. Phys. 41, 085208 (2008).

${ }^{33}$ E. György, A. Perez del Pino, P. Serra, and J. L. Morenza, Surf. Coat. Technol. 173, 265 (2003).

${ }^{34}$ J. Mori, P. Serra, E. Martianez, G. Sardin, J. Esteve, and J. Morenza, Appl. Phys. A: Mater. Sci. Process. 69, S699 (1999). 